\title{
Role of High Resolution Sonography (B-SCAN) in the Evaluation of Posterior Segment Lesions of Eye
}

\author{
Skandesh B M ${ }^{1}$, Mohan Kumar ${ }^{2}$, Sreenivasa Raju N ${ }^{3}$ \\ ${ }^{1}$ Assistant Professor, ${ }^{2}$ Assistant Professor, ${ }^{3}$ Senior Resident, Department of Radiodiagnosis, B G Nagara, Karnataka, India, \\ Adichunchanagiri Institute of Medical Sciences, B G Nagara, Karnataka, India \\ Corresponding author: Dr Mohan Kumar, 631, Kamalashree Nilaya, Balappa Layout, Bidadi Post, Ramanagar District, \\ Karnataka - 562109, India
}

DOI: 10.21276/ijcmsr.2018.3.3.3

How to cite this article: Skandesh B M, Mohan Kumar, Sreenivasa Raju N. Role of high resolution sonography (B-SCAN) in the evaluation of posterior segment lesions of eye. International Journal of Contemporary Medicine Surgery and Radiology. 2018;3(3):C11-C16.

\section{A B S T R A C T}

Introduction: High resolution ocular B-scan ultrasonography is a new tool in the quest for better diagnostic aids in the study of ocular lesion. The present study evaluates its usefulness to study various posterior segment lesions of the eye when direct ophthalmoscopy is not possible in presence of opaque media due to various causes and correlate it with the clinical findings and other investigations.

Material and methods: A prospective study was carried on 91 patients clinically suspected to have posterior segmental lesions in the presence of opaque media. Ocular ultrasonography was performed in all patients by contact method. In all cases clinical and ocular sonography diagnosis were correlated with final diagnosis to infer the accuracy.

Results: In our study trauma and infective / inflammatory etiologies accounted for most of the ocular pathologies. Vitreous (52.9\%) was the most common layer involved followed by retina and choroidal layers. Vitreous hemorrhage (31\%) was the most common vitreal abnormality and retinal detachment was most common retinal abnormality. The overall diagnostic accuracy of high resolution ocular sonography for evaluation posterior segment ocular pathologies was $98.6 \%$.

Conclusion: Ocular ultrasonography provides nonionizing, cost effective, noninvasive technique to evaluate congenital, developmental ocular anomalies, ocular tumours and trauma. B-mode ultrasonography of eye is a vital part of an ophthalmologist's diagnostic armamentarium especially with cases of opaque ocular media.

Key words: Vitreous Hemorrhage, Retinal Detachment, Opaque Ocular Media

\section{INTRODUCTION}

High resolution ultrasonography is a new tool in the quest for better diagnostic aids in the study of ocular lesion. Ultrasonography was first introduced and developed as a diagnostic tool in the field of ophthalmology, in 1950 by Dr. Karl Ossoinig. ${ }^{1}$ Henry Mundt and William Huges were the first to use one dimensional acoustic display in which echoes are represented as vertical spikes from a baseline (A-scan) in 1956. Baum and Greenwood (1958) co-developed two dimensional, brightness mode (B-scan) ultrasonography for ophthalmology in $1958 .^{2}$

Most workers prefer B-scan for ocular investigations, as it facilitates orientation and permits better recognition of tissue by two-dimensional sections of the eye. In the hands of welltrained interpreter, it provides a high degree of accuracy for ocular disorders.

Ocular ultrasonography is painless, non-invasive, safe, rapid, cost-effective, non-ionizing real time diagnostic tool and has become the most important accurate diagnostic imaging modality for directly evaluating lesions of posterior segment having opaque ocular media caused by corneal opacities, anterior chamber opacities, dense cataracts, vitreous hemorrhage, inflammatory opacities which make clinical examination and ophthalmoscopic examination difficult and least informative. ${ }^{3}$

Ultrasonography is the powerful noninvasive diagnostic tool for evaluating numerous conditions such as retinal tears, vitreous and retinal detachments, vitreous hemorrhage, sub retinal hemorrhage, eccentric disciform lesions and intra ocular tumors. ${ }^{3}$

The use of ocular ultrasonography for the evaluation of emergency patients have recently been described in the emergency medicine as a bedside procedure and it was found that it was highly accurate for ruling out and diagnosing ocular pathology in patient presenting in emergency department. $^{4}$

The visualization of orbital vasculature has opened a new dimension in ophthalmological ultrasonography exploration and vascular evaluation in normal and pathological field by the color doppler imaging which is helpful in diagnosing various pathologies like tumor, vascular malformation, fistulas and ischemic pathology. ${ }^{5}$

The present study evaluates its usefulness to study various posterior segment lesions of the eye when direct ophthalmoscopy is not possible in presence of opaque media due to various causes and correlate it with the clinical findings 
and other investigations.

\section{MATERIAL AND METHODS}

A prospective study was carried on 91 patients referred to the Department of Radiodiagnosis, Adichunchanagiri Institute of Medical Sciences, B G Nagara, for a period of 1 year from May 2017 to May 2018. Ethical clearance was taken from ethical committee of the college.

All patients clinically suspected to have posterior segmental lesions including ocular injuries in the presence of opaque media were included in the study and all cases suspected to have isolated anterior segmental and orbital lesions were excluded from the study.

Ocular ultrasonography was performed in all patients by contact method using Siemens Acuson Antares 5 ultrasonography machine equipped with 7.0 to $12.0 \mathrm{MHz}$ real time high frequency linear array transducer (probe) coupled with Color Doppler wherever necessary. The scans were usually performed with the patient supine, eye closed and directing gaze towards the ceiling. Then the probe is placed over the closed eye lid after application of coupling gel while examiner's hand rests upon the orbital margin to minimize the pressure on the globe. In all cases clinical and USG diagnosis were correlated with final diagnosis to infer the accuracy.

\section{STATISTICAL ANALYSIS}

Descriptive statistics like mean and percentages were used to calculate result.

\section{RESULTS}

The study comprised a total of 91 patients, with most of
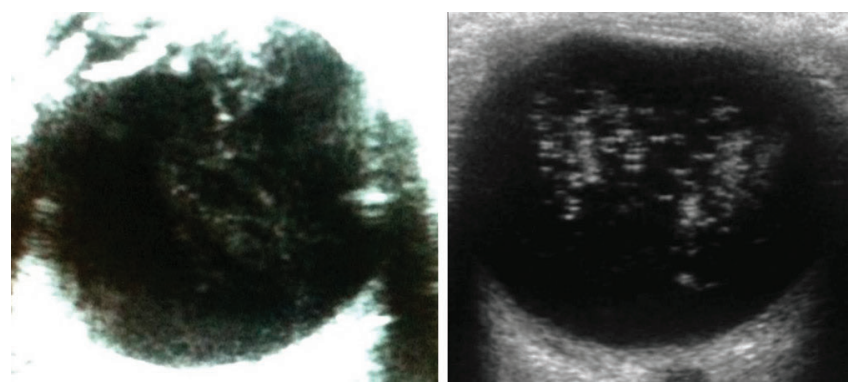

Figure-1: Vitreous detachment showing low level internal echoes with reflective membrane. Figure-2: Vitreous floaters showing uniformly distributed echoes.
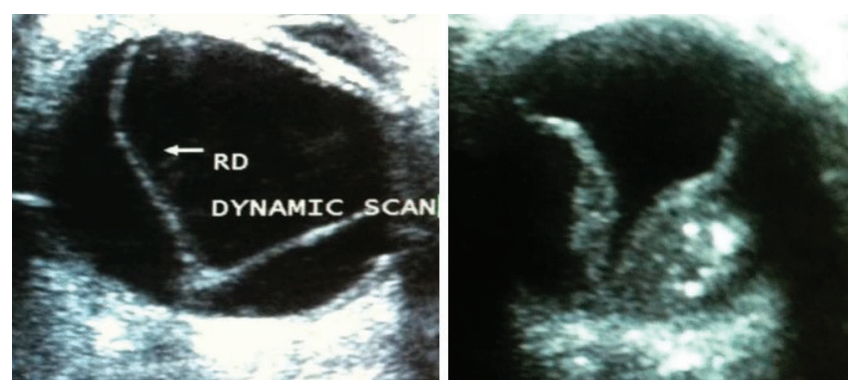

Figure-3: Retinal detachment showing folded membrane inserted into the optic disc posteriorly. Figure-4: Retinoblastoma showing echogenic mass with calcification and retinal detachment.

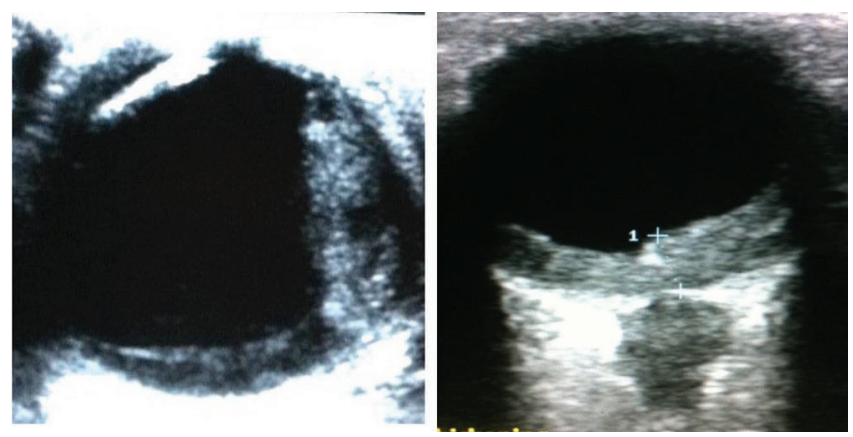

Figure-5: Choroidal granuloma dome shaped echogenic mass in the choroid. Figure-6: Choroidal metastases involving optic disc.

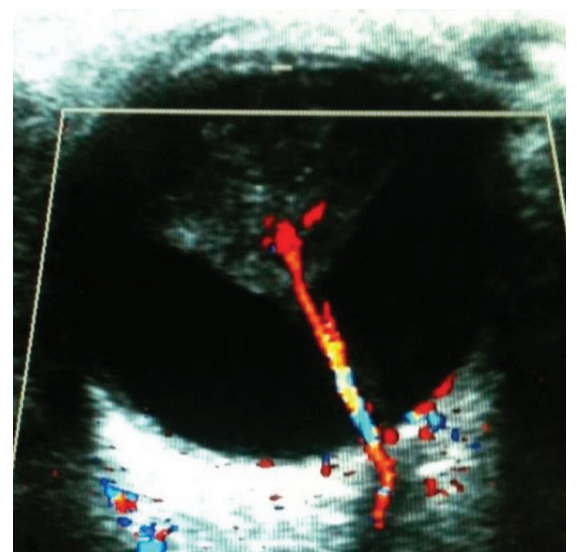

Figure-7: Persistant Hyperplastic Primary Vitreous showing retrolental membrane, a persistent hyaloid artery extending from the retrolental region to the optic disc with color flow on Doppler.

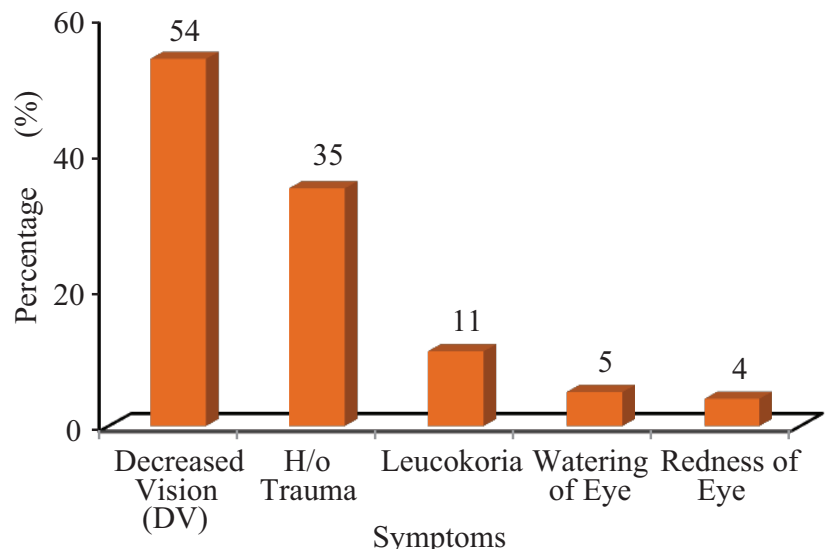

Graph-1: Showing clinical presentation

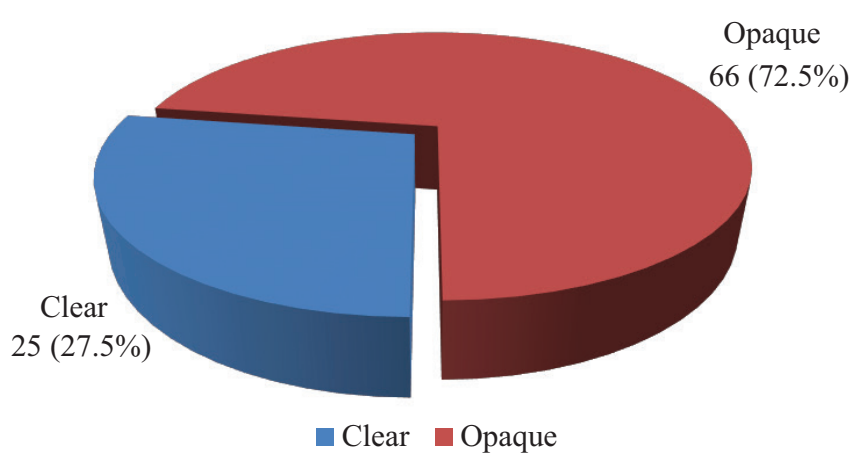

Graph-2: Showing status of media 
them belonging to age groups ranging from $31-40$ years and 41-50 years. There were 52 male and 39 female patients in the study group with male preponderance. The left eye was most commonly involved comprising $48 \%$ of cases, right eye was involved in $36 \%$ of cases and bilateral involvement was seen $16 \%$ of the cases. Majority of patients complained of decreased vision in combination with history of trauma to the eye. (Graph 1)

Out of 91 patients studied, 66 cases had opaque media and the rest 25 cases had clear media.

Trauma and infective and inflammatory etiologies accounted for most of the ocular pathologies. Vitreous hemorrhage and retinal detachment were seen in maximum number of cases who came with history of trauma. Choroidoretinal thickening was attributed to infective / inflammatory origin in majority of cases. (Graph 3)

Vitreous (52.9\%) was the most common layer involved followed by retina and choroidal layers. (Graph 4)

Vitreous hemorrhage was the most common vitreal abnormality seen in 35 cases followed by posterior vitreous detachment and vitreous floaters (Table1)

Among retinal pathologies, retinal detachment was the most common retinal abnormality seen in comprising 26 cases with retinoblastoma seen in 3 cases. Choroidal thickening

\begin{tabular}{|l|c|c|}
\hline & $\begin{array}{c}\text { No. } \\
(\mathrm{n})\end{array}$ & $\begin{array}{c}\text { Percentage } \\
(\%)\end{array}$ \\
\hline Normal & 21 & 18.6 \\
\hline Vitreous Hemorrhage (VH) & 35 & 31 \\
\hline PVD & 32 & 28.3 \\
\hline Vitreous Floaters (VF) & 10 & 8.8 \\
\hline Vitreous exudates (VE) & 8 & 7 \\
\hline Vitreous Synechiae/Bands (VB) & 4 & 3.6 \\
\hline Asteroid Hyalosis (AH) & 1 & 0.9 \\
\hline Subhyaloid hemorrhage (Sh H) & 1 & 0.9 \\
\hline PHPV & 1 & 0.9 \\
\hline Total & 113 & 100 \\
\hline \multicolumn{2}{|c|}{ Table-1: Status } \\
\hline
\end{tabular}

\begin{tabular}{|l|c|c|c|c|c|c|}
\hline & $\begin{array}{c}\text { Total } \\
\text { cases }\end{array}$ & $\begin{array}{c}\text { Ophthalmic } \\
\text { examination } \\
\text { detection }\end{array}$ & $\begin{array}{c}\text { High resolution } \\
\text { b-mode } \\
\text { ultrasnographic } \\
\text { detection }\end{array}$ & $\begin{array}{c}\text { Diagnosis } \\
\text { missed on } \\
\text { B-Scan }\end{array}$ & $\begin{array}{c}\text { \% Accuracy in } \\
\text { ophthalmic } \\
\text { examination }\end{array}$ & $\begin{array}{c}\text { \% Accuracy } \\
\text { in HRBU }\end{array}$ \\
\hline Vitreous Hemorrhage & 35 & 24 & 35 & 0 & 69 & 100 \\
\hline Posterior Vitreous Detachment & 32 & 12 & 32 & 0 & 37 & 100 \\
\hline Retinal Detachment & 26 & 11 & 25 & 1 & 39 & 96 \\
\hline Dislocated Lens in Posterior Segment & 13 & 13 & 13 & 0 & 100 & 100 \\
\hline Endophthalmitis & 12 & 9 & 12 & 0 & 75 & 100 \\
\hline Vitreous Floaters & 11 & 7 & 11 & 0 & 64 & 100 \\
\hline Neoplasm (Mass) in Posterior Segment & 5 & 5 & 5 & 0 & 100 & 100 \\
\hline Choroidal Detachment & 5 & 2 & 4 & 1 & 40 & 80 \\
\hline Scleral Perforation & 1 & 1 & 1 & 0 & 100 & 100 \\
\hline Phthisis Bulbi & 2 & 2 & 2 & 0 & 100 & 100 \\
\hline Optic Drusen & 2 & 1 & 2 & 0 & 50 & 100 \\
\hline Optic Disc Coloboma & 1 & 1 & 1 & 0 & 100 & 100 \\
\hline Papilledema & 1 & 1 & 1 & 0 & 100 & 100 \\
\hline PHPV & 1 & 1 & 1 & 0 & 100 & 100 \\
\hline & & Table-2: Type of pathology & & & \\
\hline
\end{tabular}

was seen in 12 cases (13.2\%), choroidal detachment in 5 cases (5.5\%) choroidal calcification and mass lesions were seen in 3 and 2 patients respectively. Optic disc involvement by drusen was seen in only 2 cases. Papilledema, coloboma and metastases involving optic nerve were seen in 1 case each. Among the spectrum of the posterior segment eye pathologies detected on high resolution $\mathrm{b}$-mode scan vitreous hemorrhage $(\mathrm{VH})$, posterior vitreous detachment (PVD) and retinal detachment (RD) accounted for maximum number of cases (Graph 5). In children less than 3 years of age retinoblastoma (RB) was the most common pathology.

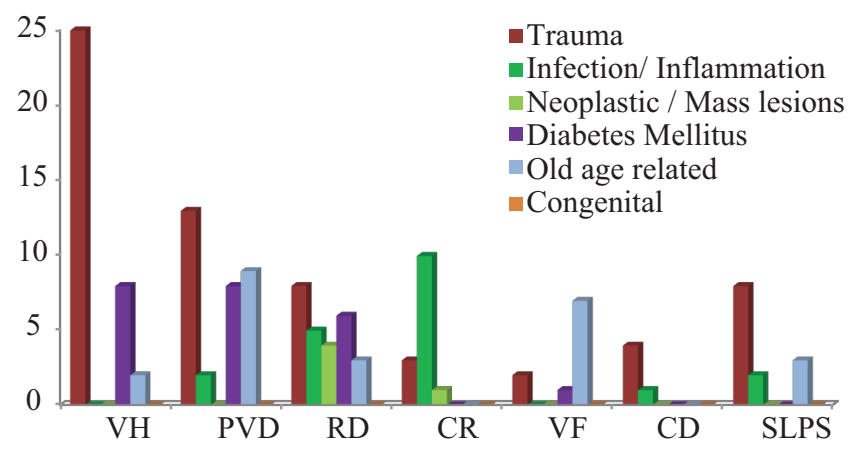

Graph-3: Etiological incidence

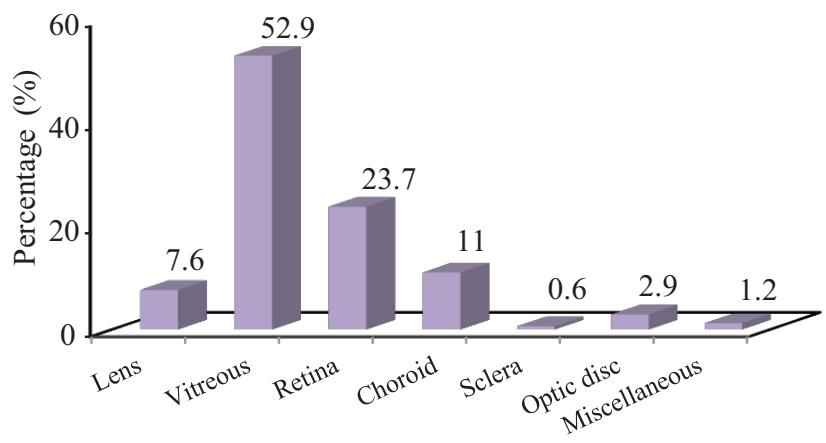

Abnormalities

Graph-4: Distribution of various ocular abnormalities 


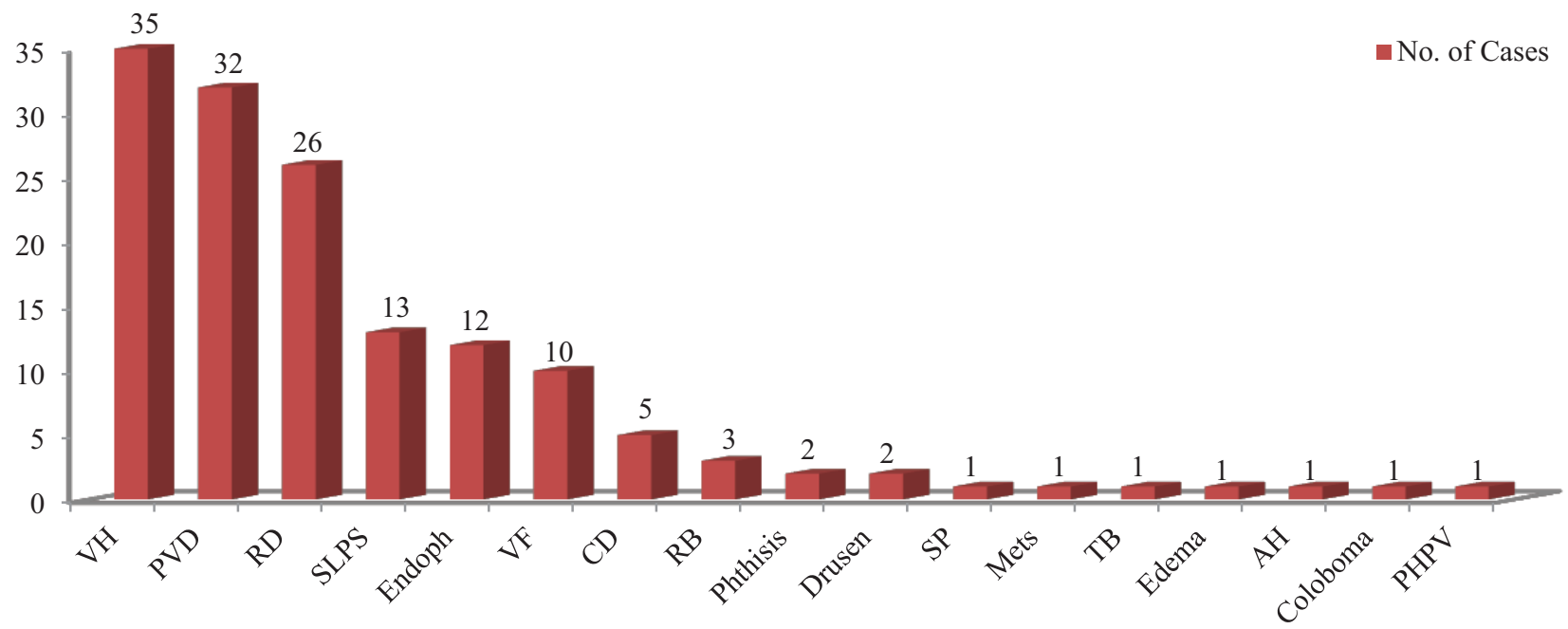

Graph-5: Pathologies of posterior segment of eye

Ophthalmic examination included visual acuity, slit lamp examination, fundoscopy, intraocular pressure measurement. Most of the cases were finally confirmed during eye surgery and cases of posterior segment tumors by CT scan and histopathology report. The diagnostic accuracy of the ocular sonography was compared with ophthalmic examination for various pathologies as mentioned in Table 2. The overall diagnostic accuracy of high resolution ocular sonography was 98.6\%.

\section{DISCUSSION}

Globe is a cystic structure and its superficial location makes it possible to study in detail the anatomy and various pathologic conditions of eye by high resolution B-mode ultrasonography. The development of high frequency, real time scanners has enhanced the diagnostic accuracy of posterior segment eye examinations in opaque ocular media. In this study 91 patients underwent ocular sonography for detection of posterior segment eye pathology especially with opaque ocular media caused by corneal opacities, anterior chamber opacities, dense cataracts, vitreous hemorrhage and inflammatory opacities seen on ophthalmic examination.

Highest number of cases was within the age group of 31-40 and 41-50 years with slight male predominance. Left eye was most commonly involved. The most presenting complaints were decreased vision (54\%) followed by trauma (35\%). Our findings correlated with study conducted by OP Sharma $(2005)^{6}$

Out of 91 cases studied 66 cases had opaque media and 25 had clear media as depicted. Cases with clear media, B scan was done either to supplement clinical assessment or to evaluate the status of retro-orbital space as described in the study conducted by Byrne et al (1986) ${ }^{7}$

Spectrum of posterior segment eye pathology detected:

Vitreous was the most common layer involved followed by retina, choroid and sclera. Common ocular pathologies detected on ocular sonography were vitreous hemorrhage, posterior vitreous detachment and retinal detachment.

\section{Vitreous hemorrhage ( $\mathrm{VH})$ :}

$\mathrm{VH}$ can occur after trauma or a retinal tear or as a complication of diabetes mellitus or a retinal vein occlusion. Fresh mild hemorrhages appear as small dots or linear areas of low reflective mobile vitreous opacities, whereas in more severe older hemorrhages, blood organizes and forms membranes (Fig 1). Vitreous hemorrhages may also layer inferiorly due to gravitational forces. ${ }^{8}$ In our study 35 cases were detected to have vitreous hemorrhage on ocular ultrasonography and was the most common abnormality seen. This correlated with study done by Sharma (2005).6,9 Trauma, proliferative diabetic retinopathy and posterior vitreous detachment were the most common causes of vitreous hemorrhage which correlate with study by Bassey Feibai et al (2011). ${ }^{10}$

Posterior Vitreous Detachment (PVD):

In our study out of 91 cases studied PVD accounted for $21.8 \%$ (32 cases) of posterior segment eye pathologies. This closely correlated with study by Jamil Ahmed et al (2009) ${ }^{9}$ $\mathrm{On} \mathrm{B-scan}$ it is seen as smooth thin freely mobile undulating membrane. On either side it is attached to ora serrata or ciliary body.

\section{Vitreous floaters:}

Floaters are due to condensations of vitreous collagen or due to blood or glial tissue torn from an area adjacent to the optic nerve head. In our study 10 cases were detected to have vitreous floaters. It accounted for $8.8 \%$ of the total vitreous abnormalities which closely correlated with study by Zafar Dawood et al (2008). ${ }^{11}$ Vitreous floaters were seen as few scattered low intensity echoes.

\section{Retinal detachment:}

Seen as bright continuous thin folded membrane which inserted into the optic disc posteriorly and ora serrata in the periphery (Fig 3). On kinetic echography this membrane showed very minimal to restricted after eye movements.

In our study out of 91 cases studied 26 cases (28.6\%) had retinal detachment. This closely correlated with study by OP Sharma (2005). ${ }^{6} 1$ case was missed on B-scan due to complex vitreous echoes. This was detected during ocular surgery. This correlated with study by Jose LC et al (2009). ${ }^{12}$ Trauma and diabetes were the most common causes of retinal detachment. 


\section{Retinoblastoma}

Retinoblastoma is the most common intraocular malignancy in children. It is seen as mass in the posterior segment, with calcification and vascularity was observed on color Doppler. (Fig 4)

In our study out of 91 cases, 3 cases were diagnosed to have retinoblastoma by HRBU who were within 3 years of age. This correlated with study by Suryasnata R (2010). ${ }^{13} 1$ case (33\%) had bilateral affection with retinoblastoma arising from posterior segments of both eyes. Study by Suryasnata $\mathrm{R}(2010)^{13}$ on Indian population showed a slightly higher incidence of bilateral tumors $45.8 \%$.

Retinochoroidal thickening:

The main contributing factor was ocular inflammation and as a sequel of trauma to the eye. On B-mode USG low reflective dot like vitreous echoes with thickening of retinochoroidal layer were seen suggestive of vitreous inflammation. This corresponded to study by Chan IM et al (1984). ${ }^{14}$ In our study retinochoroidal thickening accounted for $8.1 \%$ (12 cases) posterior segment eye pathologies.

Choroidal detachment:

As fluid accumulates in suprachoroidal space the choroid is displaced from its normal position. Serous choroidal detachment involves transudation of serum into the suprachoroidal space. Hemorrhagic choroidal detachment is a hemorrhage in the suprachoroidal space or within the choroid caused by the rupture of choroidal vessels. On B-mode USG choroidal detachment was seen as smooth, thick, dome shaped membrane in the periphery with no after movements.

Choroidal detachment accounted for $3.4 \%$ of posterior segment pathology in the present study which correlated with study by Ferrer E et al (2013) ${ }^{15}$ in which choroidal detachment accounted for $3.8 \%$ of posterior segment pathology. In our study out of 91 cases; 5 cases were associated with choroidal detachment. 4 cases (80\%) were detected to have CD by HRBU. Diagnosis of 1 case was missed by B-mode USG. This was detected during eye surgery.

\section{Choroidal tuberculoma}

Amelanotic choroidal granuloma could be of infectious or inflammatory origin such as tuberculosis and sarcoidosis. It may mimic choroidal melanoma or metastasis. Delay in detecting this treatable condition can have serious consequences on the patient's eye. ${ }^{16}$ In the present study 1 case was detected to have choroidal tuberculoma. The patient came with complaint of dimness of vision for 2 weeks. On USG dome shaped echogenic mass was noted in the choroid in the posterior pole (Fig 5). Associated exudative retinal detachment was noted. Chest $X$ ray revealed tubercular infection which was confirmed by sputum examination. Diagnostic paracentesis of aqueous humor revealed a raised Anti IgM titre for mycobacterium. On initiation of antitubercular treatment the lesion resolved into scar after 10 weeks with partial improvement of vision.

\section{Choroidal metastases:}

Single case of bilateral choroidal metastases involving optic disc and optic nerve was noted in our study in a case of renal cell carcinoma with lung metastases. On USG, mass lesions were noted involving bilateral posterior choroids with thickening of optic nerve including optic disc (Fig 6) and it was associated with total retinal detachment. Renal cell carcinoma is its ability to metastasize to unusual sites including the gall bladder, pancreas, myocardium, and occasionally the eye and orbit. ${ }^{17}$ Shields and colleagues reported that the most common site of involvement was the choroid (88\%), followed by the iris (9\%), and the ciliary body (2\%). The origin of metastatic foci to the uvea is most often from breast carcinoma in women and lung carcinoma in men, with kidney malignancies representing only $2 \%$ of cases overall. ${ }^{18}$

\section{Optic disc drusen:}

In our study 2 cases were seen on ocular sonography. Scanning demonstrated a collection of calcific material in the optic nerve head, sometimes protruding into the vitreous and causing acoustic shadow. Congenital drusens are asymptomatic but hemorrhage / neovascularization can occur resulting in visual impairment and are also manifestations of age related macular degeneration. Many elderly patients with drusen have normal vision but a significant number of patients develop progressive impairment in central vision. ${ }^{19}$

\section{Optic disc coloboma}

Colobomas are congenital or acquired notches, fissures, or defects that are found in the eye. Most commonly, they are congenital and occurs secondary to faulty closure of the embryonic fissure. The optic nerve alone may be involved, or, more often, the anomaly may be of the retinochoroidal variety. Isolated optic disc coloboma appears as excavation within the nerve head. They may be unilateral or bilateral. In our study among 91 cases 1 case was diagnosed to have optic disc coloboma with involvement of the adjacent choroid without affecting anterior segment. This was a 10 years male patient who came with history of redness of right eye and decreased vision of right eye. Fundoscopy of right eye revealed defect in the optic disc and the choroid.

\section{Persistant Hyperplastic Primary Vitreous (PHPV)}

$\mathrm{PHPV}$ is a serious unilateral disorder of the vitreous due to failure of regression of the primary vitreous. B-scan shows a retrolental membrane, which may be dense; there is a persistent hyaloid artery extending from the retrolental region to the optic disc. ${ }^{20}$ In our study 1 case was seen in 1 year old boy who came with leucocoria in right eye showing dense retrolental high reflective vitreous membrane extending from the posterior lens capsule to the optic disc. On color doppler there was e/o color flow within the membrane suggestive of persistent hyaloid vessel (Fig 7).

\section{Dislocated lens}

On B-scan the dislocated lens is seen as elliptical shaped structure of varying reflectivity situated in the vitreous cavity with few of them lying on the retinal surface. On eye movements the dislocated lens will change its position in the vitreous cavity. In our study totally 13 cases were detected with dislocated subluxated lens (8.7\%). This closely correlated with study by OP Sharma (2005). ${ }^{6}$

\footnotetext{
Scleral perforation

On USG, it is seen as defect in sclera with prolapse of vitreous
} 
and uveal tissue through the defect. Associated vitreous hemorrhage may be noted. One case of scleral perforation resulting from traumatic open globe injury was noted in our study.

Diagnostic accuracy of high resolution b-mode ultrasonography in our study:

Out of 147 pathologies detected 2 were missed on B-scan (1 case each of retinal detachment and choroidal detachment). The accuracy of B-scan in evaluation of posterior segment lesions in our study was calculated to be $98.6 \%$. This corresponds to study by Ejaz AJ et al (2007) ${ }^{21}$

\section{CONCLUSION}

Ocular ultrasonography with high frequency probes provide nonionizing, cost effective, noninvasive technique which is easily available and can be performed in outdoor patient without any use of anesthetics or sedative therapy. B-mode ultrasonography of eye is a vital part of an ophthalmologist's diagnostic armamentarium especially with cases of opaque ocular media. B-scan USG is highly sensitive to evaluate congenital, developmental anomalies, ocular tumours and trauma. Keeping all these points in view we can safely conclude that " $B$-scan a diagnostic tool in ocular pathologies".

\section{REFERENCES}

1. Ossoinig KC.Standardized echography: Basic principles, clinical applications, and results. Int Ophthalmol Clin 1979;19(1):127-210.

2. Baun $G$, Greenwood I. The application of ultrasonic locating techniques to ophthalmology. Arch ophthalmol 1958; 60(2): 263-279.

3. Brandy $\mathrm{CH}$, Linda K, Arun DS. Ophthalmic ultrasonography: Theoretic and practical considerations. Ultrasound clinics. [online] 2008; 3 (2):179-83.

4. Blaivas M, Theodoro D, Sierzenski PR. Acad Emeg Med. 2002;9(8): 791-9.

5. Georgeta M, Al A, Florica B. Color Doppler echography in oculo-orbital diseases. Oftalmologia. 2001; 52 (2): 21-9.

6. Sharma OP. Orbital sonography with it's clinicosurgical correlation. Indian J Radiol Imaging 2005; 15(4):537-54.

7. Byrne SF, Green RL: Ultrasound of eye and orbit, Library of Congress cataloging in publication data. In Mosby year book 1992; chapter 4: 95-131.

8. Waldron RG, Aaberg TM. B-scan ocular ultrasound. Emedicine from web MD 2012 Feb: Available from emedicine.medscape.com/article 1228865

9. Ahmed J, Shaikh FF, Rizwan A, Memon MF. Evaluation of Vitreo-Retinal Pathologies Using B-Scan.Ultrasound Pak J Ophthalmol 2009; 25(4):7-12.

10. Bassey Fiebai, Chinyere Nnenne Pedro-Egbe. Cause of Vitreous Hemorrhage In Port Harcourt. The Nigerian Health Journal. 2011: 11 (2):67-68.

11. Zafar D, Sajad AM, Qadeer A: Role of B-Scan Ultrasonography for Posterior Segment Lesions. JLUMHS 2008; 7(1):7-12.

12. Lorenzo-Carrero J, Perez-Fbores I, Monica CidGalano $\mathrm{M}$ et al. B-scan ultrasonography to screen for retinal tears in acute symptomatic age-related posterior vitreous detachment. Ophthalmology 2009;116(1):9499.

13. Suryasnata R, Ghanashyam B, Geeta KV, Santosh H, Vijay AR. Retinoblastoma: Presentation, Treatment and Outcome at A Tertiary Eye Care Center in Eastern India. AIOC 2010:517-518.

14. Chan IM, Jalkh AE, Trempe CL, Tolentino FI. Ultrasonographic finding in Endophthalmitis. Ann Ophthalmol. 1984; 16 (8): 778-784.

15. Ferrer E, Mendoza R, Dessi G, Stefanini T, Spezia L, Zaragoza. Role of B-scan ocular ultrasound as adjuvant for the clinical assessment of eyeball diseases. Poster No.:C-1323, ECR Congress 2013: 1-22.

16. Ahmad MA, Hassan A, Hani SA, Ahmed MA. Solitary presumed choroidal tuberculomas masquerading as choroidal tumors Saudi Med J 2013; 34 (1): 86-90.

17. Shome D, Honavar SG, Gupta $\mathrm{P}$ et al. Metastasis to the eye and orbit from renal cell carcinoma. A report of three cases and review of literature. Surv Ophthalmol. 2007; 52(2):213-223.

18. Shields CL, Shields JA, Gross NE, et al. Survey of 520 eyes with uveal metastases. Ophthalmology 1997; 104(3): 1265-76.

19. Smitty WE, Fine SL. Prognosis in patients with bilateral macular drusen. Opthalmology 1984; 91(5): 271-277.

20. David Sutton. David Sutton's Textbook of Radiology and imaging: Vol 2. $7^{\text {th }}$ ed. Elsevier Ltd. 2011: 1557-58.

21. Ejaz AJ, Aamir AC, Iftikhar A, Mehmood $\mathrm{H}$. Diagnostic Applications of "B Scan" Pak J Ophthalmol 2007; 23(2):80-83.

Source of Support: Nil; Conflict of Interest: None

Submitted: 27-06-2018; Accepted: 17-07-2018; Published online: 28-07-2018 